\title{
$\beta$-Shaped intracorporeal Roux-en-Y reconstruction after totally laparoscopic distal gastrectomy
}

\author{
Kazuo Motoyama $\cdot$ Kazuyuki Kojima $\cdot$ \\ Mikiko Hayashi · Keiji Kato • Mikito Inokuchi • \\ Kenichi Sugihara
}

Received: 8 March 2013/Accepted: 5 October 2013/Published online: 1 November 2013

(c) The International Gastric Cancer Association and The Japanese Gastric Cancer Association 2013

\begin{abstract}
Background The use of laparoscopic gastrectomy for the treatment of gastric cancer has been increasing. Roux-en-Y (R-Y) reconstruction after laparoscopy-assisted distal gastrectomy is now widely used to decrease leakage and prevent reflux. Owing to the need for a less invasive technique, we have developed a new technique for intracorporeal $\mathrm{R}-\mathrm{Y}$ reconstruction ( $\beta$ reconstruction) after totally laparoscopic distal gastrectomy (TLDG).

Methods In this report, we describe the $\beta$ reconstruction technique and short-term outcomes of the initial 105 patients who underwent $\beta$ reconstruction from December 2008 to March 2012.

Results The operative and $\beta$ reconstruction times were $330 \pm 61.3$ and $29 \pm 5.6$ min (mean \pm SD), respectively. Anastomotic leakage after gastrojejunostomy occurred in one patient $(0.9 \%)$, requiring reoperation. Four cases $(3.8 \%)$ of anastomotic stenosis required endoscopic balloon dilation. However, R-Y stasis was not noted.
\end{abstract}

K. Motoyama $(\bowtie) \cdot$ K. Kojima $\cdot$ M. Hayashi · K. Kato ·

M. Inokuchi

Department of Esophageal and Gastric Surgery, Tokyo Medical and Dental University, Tokyo, Japan

e-mail: motoyama@kb3.so-net.ne.jp

K. Motoyama - M. Hayashi · K. Kato · M. Inokuchi ·

K. Sugihara

Department of Surgical Oncology, Graduate School of Medical and Dental Sciences, Tokyo Medical and Dental University,

Tokyo, Japan

K. Kojima

Center for Minimally Invasive Surgery, Tokyo Medical and

Dental University, Tokyo, Japan
Conclusions We have indicated a technical description as well as the usefulness of $\beta$-shaped intracorporeal $\mathrm{R}-\mathrm{Y}$ reconstruction after TLDG.

Keywords Gastric cancer - Totally laparoscopic distal gastrectomy $\cdot$ Roux-en-Y reconstruction

\section{Introduction}

Gastric cancer remains one of the most common forms of cancer worldwide, with an estimated 989,600 new cases and 738,000 deaths per year in 2008, making it the second highest cause of cancer-related deaths [1, 2]. Gastric cancer is more prevalent in Japan than in Western countries [2], and early gastric cancer accounts for nearly $60 \%$ of all cases of gastric cancer; this increased incidence can partly be attributed to Japan's nationwide mass screening programs for gastric cancer [3]. Consequently, the demand for minimally invasive treatments of early gastric cancer has been increasing and warrants the development of new therapeutic methods and modalities.

Distal gastrectomy (DG) is performed on patients with gastric cancer located in the lower two-thirds of the stomach. Since laparoscopy-assisted distal gastrectomy (LADG) for early gastric cancer was first described in 1994 [4], it has become a common surgical option for gastric cancer in Japan [5, 6]. Subsequent reconstruction after DG is usually performed by using either Billroth I (B-I) or II (B-II) (in Japan and Korea), or Roux-en-Y (R-Y) reconstruction (the preferred option in Western countries).

Recently, owing to the decreased leakage rates and bile reflux, R-Y reconstruction has been increasingly used after DG in Japan. We have previously reported on the advantages of R-Y anastomosis in LADG [7-9]. However, 
patient size and therefore access for an anastomosis through an epigastric minilaparotomy (4-6 cm in size) may cause technical difficulties. Hence, to avoid this problem, we have developed a novel technique for a safer intracorporeal R-Y reconstruction, the so-called $\beta$ reconstruction.

This new technique offers a wide field of view, with ample access and space to create the anastomosis. In this report, we describe the technical details of $\beta$ reconstruction and short-term outcomes of the initial 105 consecutive procedures.

\section{Methods}

From December 2008 to March 2012, a total of 105 patients with histopathologically diagnosed gastric cancer underwent totally laparoscopic distal gastrectomy (TLDG) at the Department of Esophageal and Gastric Surgery, Tokyo Medical and Dental University. Age and sex were recorded for every case. Body mass index (BMI) was calculated using preoperative physical measurements. The preoperative staging performed for all the patients involved gastroendoscopy, endoscopic ultrasonography and computed tomography.

Intraoperative data included operative time, $\beta$ reconstruction time, blood loss volume and extent of lymph node dissection. The extent of lymph node dissection was decided according to clinical stage (classified according to the third English edition of the Japanese classification of gastric carcinoma [10] and third version of the 2010 Japanese gastric cancer treatment guidelines [11]). Postoperative outcomes were measured by several parameters: when clear liquids were first administered, when a soft diet was commenced and length of postoperative hospital stay. A postoperative complication was defined as any event requiring specific medical or surgical treatment. Other factors included pancreatitis, wound infection, intraabdominal bleeding, ileus and enterocolitis.

\section{Surgical technique for $\beta$-shaped intracorporeal R-Y reconstruction}

Each patient was placed in the reverse Trendelenburg position under general anesthesia. A 12-mm trocar was inserted at the umbilical incision. The remaining four ports were set up, involving two 5-mm incisions in the right and left upper quadrants, and two 12-mm incisions in the right and left lower quadrants. A Nathanson liver retractor was inserted just below the xiphoid to retract the liver.

Mobilization of the stomach and en bloc lymph node dissection were performed as described previously [7, 9]. A seromuscular suture with laparoscopic hand-sewn

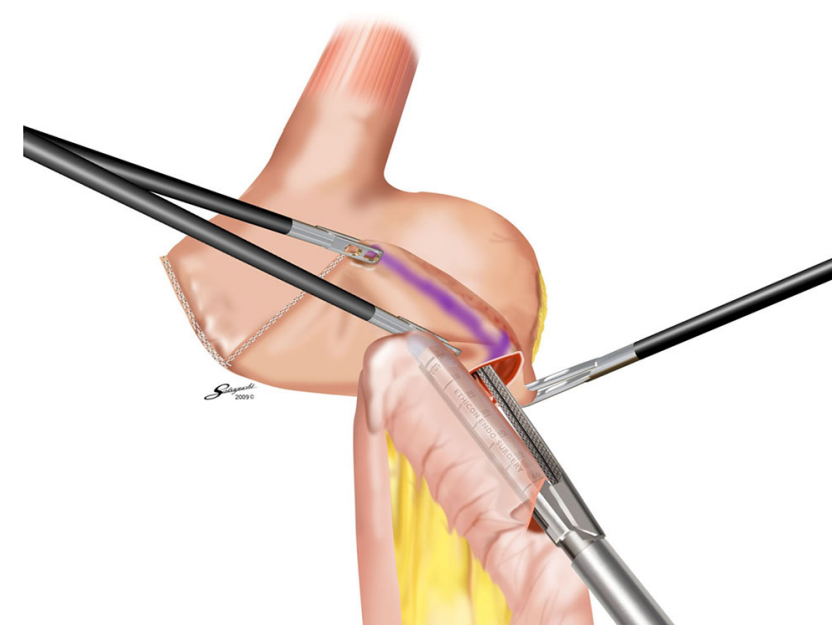

Fig. 1 An isoperistaltic gastrojejunostomy was constructed by a sideto-side anastomosis

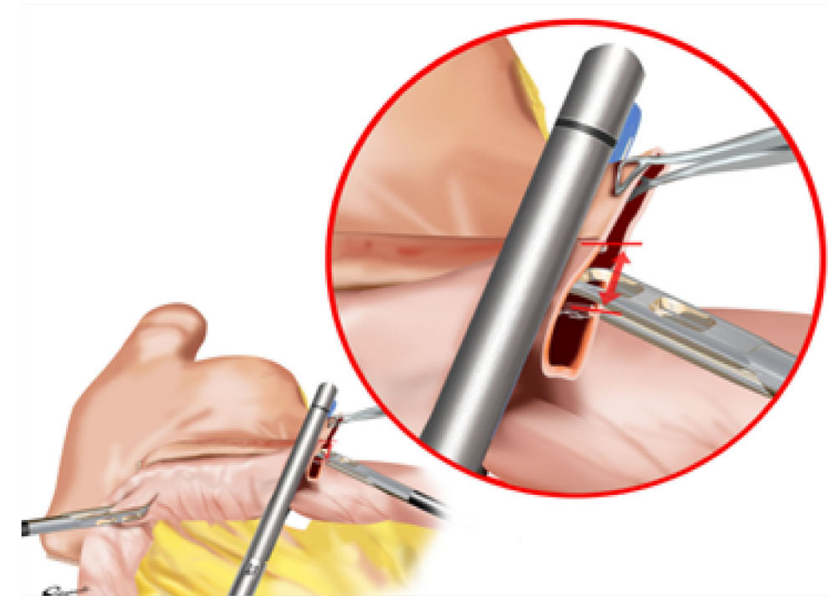

Fig. 2 The common entry hole of the gastrojejunostomy and the jejunum were closed together

technique was used for the duodenal stump after excision of the duodenum.

A purple dye was used to mark the proximal end of the resection site on the anterior wall of the stomach. An endoscopic linear stapler (Echelon Flex 60 with a 60/3.8mm gold cartridge, Ethicon, Cincinnati, OH, USA) was used to dissect the proximal side of the stomach; the dissected stomach was then externalized through the umbilical incision, which was extended to approximately $4 \mathrm{~cm}$.

After reestablishing a pneumoperitoneum, a small incision was made on the greater curvature of the remnant stomach. The antimesenteric surface of the jejunum $25 \mathrm{~cm}$ distal to the ligament of Treitz was marked with a purple dye, at which point a small incision was made. The cartridge fork of the endoscopic linear stapler was inserted through the small incision of the jejunum, and the jejunal loop was brought up the antecolic route. An isoperistaltic gastrojejunostomy was performed, creating a side-to-side 
anastomosis using the Echelon Flex 60 with a 60/3.5-mm blue cartridge (Fig. 1).

A small hole was made on the jejunal mesentery to insert the stapler. The first assistant surgeon carefully held the anterior and posterior sides of the staple line of the gastrojejunostomy, ensuring that there was no overlap. The common entry hole for the gastrojejunostomy and the jejunal loop were closed together with a single Echelon Flex 60 stapler with a $60 / 3.5-\mathrm{mm}$ blue cartridge (Fig. 2). The closure of the entry hole and dissection of the jejunal loop were performed simultaneously by the stapler, resulting in a functional end-to-end anastomosis between the stomach and jejunum, which was completed intracorporeally. The staple lines were laparoscopically inspected for bleeding.

Finally, a jejunojejunostomy was performed extracorporeally to save time by using the 4-cm umbilical incision from which the resected stomach was removed for normal patients. However, we needed to perform the jejunojejunostomy intracorporeally in the case of severely obese patients. Closure of the side-to-side anastomosis between the jejunum $(30 \mathrm{~cm}$ distal to the site of the gastrojejunostomy) and proximal loop of the jejunum stump was performed using Echelon Flex 60 with a 60/2.5-mm white cartridge. The common entry hole was closed with another linear stapler. The closure of the mesentery gap and Petersen's space $[12,13]$, known to be vulnerable to defects

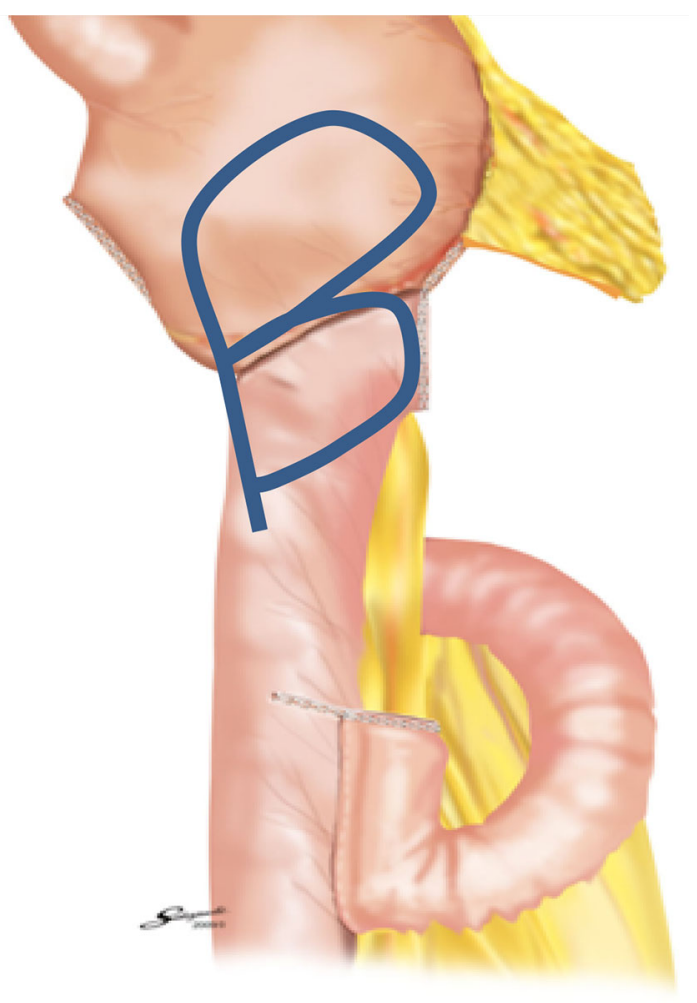

Fig. 3 The $\beta$-shaped intracorporeal R-Y reconstruction was completed resulting from R-Y reconstruction, was performed to prevent internal herniation. This completed the $\beta$-shaped intracorporeal R-Y reconstruction (Fig. 3).

\section{Results}

From December 2008 to March 2012, we performed $\beta$ shaped intracorporeal R-Y reconstruction after TLDG for gastric cancer in 105 consecutive patients ( 74 men and 31 women). The characteristics of the patients are shown in Table 1. The mean patient age was $66 \pm 12$ years (mean $\pm \mathrm{SD}$ ), and the mean BMI was $23 \pm 2.7 \mathrm{~kg} / \mathrm{m}^{2}$ (mean $\pm \mathrm{SD}$ ). The maximum BMI in our series was $29.5 \mathrm{~kg} / \mathrm{m}^{2}$. In the present study, there was no relationship between BMI and complications. Most of the patients $(74.3 \%)$ had stage IA gastric cancer according to the pathological staging of gastric cancer.

Table 1 Patients' characteristics

\begin{tabular}{ll}
\hline No. of patients & 105 \\
Sex & \\
Male & 74 \\
Female & 31 \\
Age, mean \pm SD (years) & $66 \pm 12$ \\
BMI, mean \pm SD $\left(\mathrm{kg} / \mathrm{m}^{2}\right)$ & $23 \pm 2.7$ \\
Pathological tumor stage & \\
IA & $78(74.3 \%)$ \\
IB & $15(14.3 \%)$ \\
IIA & $7(6.7 \%)$ \\
IIB & $2(1.9 \%)$ \\
IIIA & $1(0.9 \%)$ \\
IIIB & $2(1.9 \%)$ \\
\hline
\end{tabular}

Table 2 Intraoperative data and postoperative outcomes

\begin{tabular}{ll}
\hline Operative time, mean $\pm \mathrm{SD}(\mathrm{min})$ & $330 \pm 61.3$ \\
$\beta$ Reconstruction time, mean $\pm \mathrm{SD}(\mathrm{min})$ & $29 \pm 5.6$ \\
Blood loss volume, mean $\pm \mathrm{SD}(\mathrm{min})$ & $88 \pm 91$ \\
Extent of lymph node dissection & \\
$\mathrm{D} 1$ & $2(1.9 \%)$ \\
$\mathrm{D} 1+8 \mathrm{a}$ & $1(0.9 \%)$ \\
$\mathrm{D} 1+8 \mathrm{a}, 9$ & $88(83.8 \%)$ \\
$\mathrm{D} 1+8 \mathrm{a}, 9,11 \mathrm{p}$ & $1(0.9 \%)$ \\
D1 $+8 \mathrm{a}, 9,12 \mathrm{a}$ & $1(0.9 \%)$ \\
D2 & $12(11.4 \%)$ \\
Clear liquids initiated, median (days) & 1 \\
Soft diet initiated, median (days) & 2 \\
Postoperative hospital stay, median (days) & 7
\end{tabular}

D1 1, 3, 4sb, 4d, 5, 6, 7

D2 1, 3, 4sb, 4d, 5, 6, 7, 8a, 9, 11p, 12a 
Table 3 Postoperative complications

\begin{tabular}{ll}
\hline Anastomotic leakage (gastrojejunostomy) & $1(0.9 \%)$ \\
Anastomotic stenosis (gastrojejunostomy) & $4(3.8 \%)$ \\
Anastomotic bleeding & $0(0 \%)$ \\
R-Y stasis & $0(0 \%)$ \\
Internal hernia & $2(1.9 \%)$ \\
Intraabdominal abscess $^{\text {Others }}{ }^{\mathrm{a}}$ & $1(0.9 \%)$ \\
\hline
\end{tabular}

${ }^{a}$ Pancreatitis, wound infection, intra-abdominal bleeding, ileus, enterocolitis

The intraoperative data and postoperative outcomes are summarized in Table 2. The operative time was $330 \pm 61.3$ min (mean $\pm \mathrm{SD}$ ). The $\beta$ reconstruction time was $29 \pm 5.6 \mathrm{~min}$ (mean $\pm \mathrm{SD}$ ). The blood loss volume was $88 \pm 91 \mathrm{ml}$ (mean $\pm \mathrm{SD}$ ). After TLDG, on average, the patients tolerated liquids on the first day and a soft diet on the second postoperative day. The median postoperative hospital stay was 7 days.

The postoperative complications are summarized in Table 3. Overall, anastomotic leakage after gastrojejunostomy occurred in one patient $(0.9 \%)$, requiring reoperation on the second postoperative day. Hence, laparoscopic intraperitoneal drainage and closure of the gastrojejunal leakage were performed. Four patients $(3.8 \%)$ had anastomotic stenosis, of whom three were treated using endoscopic balloon dilation and one was treated conservatively. No cases of functional stasis after R-Y reconstruction were noted in this study. An intra-abdominal abscess was noted in one patient $(0.9 \%)$, requiring image-guided drainage. There was no conversion to open surgery in any of the patients.

\section{Discussion}

LADG has become one of the standard treatment options for gastric cancer in Japan. B-I reconstruction is usually performed after LADG [14, 15], as the gastroduodenostomy involves the creation of only a single anastomosis, thus reducing reconstruction time. Moreover, B-I reconstruction has the physiological advantage of permitting the passage of food through the duodenum [16]. However, compared to B-I reconstruction, R-Y reconstruction also has certain advantages, such as a reduction in the incidence of anastomotic leakage and prevention of remnant gastritis and bile reflux into the remnant stomach [7, 8, 17-21]. Hence, R-Y reconstruction after LADG has been the firstchoice reconstruction technique at our institution since March 2003.

In $\mathrm{R}-\mathrm{Y}$ reconstruction after LADG, gastrojejunostomy is performed by elevating the remnant stomach and jejunal stump via epigastric minilaparotomy under direct vision. However, we noted that it is very difficult to perform a gastrojejunostomy via a minilaparotomy in severely obese patients with a thick abdominal wall, even if the small incision is extended. Park et al. [22] reported that $7 \%$ (21/ 300 ) of patients experienced postoperative wound infection after LADG. In R-Y reconstruction after TLDG, the gastrojejunostomy is performed intracorporeally. Song et al. [23] reported that the bowel recovery time was shorter in TLDG than in LADG. Moreover, Kim et al. [24] reported that TLDG contributed to the improvement of early surgical outcomes, including bowel movement, pain score, overall complication rate and time until hospital discharge. Therefore, we have implemented our newly developed technique for intracorporeal $\mathrm{R}-\mathrm{Y}$ reconstruction after TLDG in our institution since December 2008. We have termed this procedure as " $\beta$-shaped intracorporeal R-Y reconstruction ( $\beta$ reconstruction)" because of its similarity to the $\beta$ character (Fig. 3).

In the present study, the operating time in the 105 patients treated by TLDG $+\mathrm{R}-\mathrm{Y}$ ( $\beta$ reconstruction) was $330 \mathrm{~min}$; moreover, the morbidity-related problems among these patients included anastomotic leakage in 1/105 cases $(0.9 \%)$ and anastomotic stenosis in $4 / 105$ cases $(3.8 \%)$. In the Japanese Phase II trial (JCOG0703) that confirmed the safety and feasibility of LADG for the treatment of clinical stage I gastric cancer, the operating time was $250 \mathrm{~min}$, and anastomotic leakage and anastomotic stenosis were observed in $3 / 176$ cases $(1.7 \%)$ and $1 / 176$ cases $(0.6 \%)$, respectively [5]. In the JCOG0703, reconstruction was performed extracorporeally and the reconstruction method involved B-I, R-Y and gastro-gastrostomy (pylorus-preserving distal gastrectomy). In the present study, reconstruction was performed intracorporeally, and the reconstruction method involved only R-Y. Although the findings of the JCOG0703 cannot be compared with those of the present study for the above-mentioned reason, we noted that the TLDG+R-Y ( $\beta$ reconstruction) used in the present study has a longer operating time and lower incidence of anastomotic leakage and greater incidence of anastomotic stenosis, as compared to the technique used in the JCOG0703. In a previous study, we noted that the operating time and morbidity-related problems in patients treated with $\mathrm{LADG}+\mathrm{R}-\mathrm{Y}$ were $275 \mathrm{~min}$ and anastomotic stenosis in $1 / 68$ cases $(1.5 \%)$ with no leakage, respectively [7]. The operating time of TLDG $+\mathrm{R}-\mathrm{Y}$ ( $\beta$ reconstruction) is longer than that required for the technique used in the JCOG0703 or for LADG+R-Y. This is partly because the number of surgeons undergoing training has increased since the introduction of the $\beta$ reconstruction technique; therefore, the time required for lymph node dissection was longer. The time required for $\beta$ reconstruction in the $\mathrm{TLDG}+\mathrm{R}-\mathrm{Y}$ procedure is approximately $30 \mathrm{~min}$, which is 
almost the same as that required for reconstruction after LADG. We believe that TLDG is superior to LADG as the patient size does not affect the ease of performing the gastrojejunostomy procedure. Furthermore, a greater incidence of anastomotic stenosis was noted in TLDG+R-Y ( $\beta$ reconstruction) than in the procedure used in the JCOG0703 or in LADG+R-Y; the four cases of anastomotic stenosis observed following TLDG $+R-Y$ ( $\beta$ reconstruction) had developed during the early stages after the introduction of $\beta$ reconstruction, and this complication has not been observed recently. This is partly because of improvements in the surgical procedure, including the care taken to avoid excessive compression of the jejunum as well as avoiding forced closure of the stapler while sealing the common entry hole and jejunum in order to prevent anastomotic stenosis. We believe that the incidence of anastomotic stenosis will decrease as experience with this technique is accumulated.

A characteristic feature of $\beta$ reconstruction is that intracorporeal suturing is not required. This is an important issue as hand-suturing is difficult and time-consuming for certain surgeons. This is also a feature of intracorporeal $\mathrm{R}-\mathrm{Y}$ reconstruction with a gastrojejunal antiperistaltic anastomosis, as described by Takaori et al. [25] and Bouras et al. [26], thus distinguishing this procedure from $\beta$ reconstruction with a gastrojejunal isoperistaltic anastomosis. They stated that intracorporeally stapled R-Y reconstruction after TLDG can prevent the twisting and torsion of the jejunal loop, and we believe that these benefits also apply to intracorporeal reconstruction. However, laparoscopically assisted uncut R-Y gastrojejunostomy [27] requires intracorporeal suturing, thus distinguishing this procedure from $\beta$ reconstruction.

Another characteristic feature of $\beta$ reconstruction is that it facilitates the sealing of the common entry hole of gastrojejunostomy and division of the jejunum simultaneously by using one stapler; this simultaneous procedure can be successfully performed in $90 \%$ of cases. However, in cases where a small incision that is made on the remnant stomach becomes larger and when the jejunum is excessively compressed, we believe that two staplers should be used to prevent anastomotic stenosis.

In a previous study on 68 patients treated with $\mathrm{LADG}+\mathrm{R}-$ Y, no development of R-Y stasis was noted [7]. Based on the findings of that study, we decided to perform isoperistaltic $\mathrm{R}-\mathrm{Y}$ reconstruction after TLDG. Uyama et al. intracorporeally performed 42 laparoscopically assisted uncut R-Y gastrojejunostomy procedures [28-31] in order to prevent development of R-Y stasis [32, 33]; they reported only one problem with this uncut R-Y operation, which involved the frequent recanalization of the stapled occlusion of the afferent loop [34-36]. In the present study, we did not observe the development of postoperative R-Y stasis in any of the 105 patients. This is partly because we selected a Roux limb length of approximately $25-30 \mathrm{~cm}$ to permit a certain extent of expansion of the jejunum, as several studies reported that very long Roux limbs cause R-Y stasis.

In conclusion, in the present report, we have indicated a technical description as well as the usefulness of $\beta$-shaped intracorporeal R-Y reconstruction after TLDG and provided the short-term outcomes of the initial 105 patients who underwent this procedure. In a further study, we will assess the long-term postoperative results of $\beta$ reconstruction.

Conflict of interest Drs. Motoyama, Kojima, Hayashi, Kato, Inokuchi and Sugihara have no conflicts of interest or financial ties to disclose.

\section{References}

1. Jemal A, Bray F, Center MM, Ferlay J, Ward E, Forman D. Global cancer statistics. CA Cancer J Clin. 2011;61:69-90.

2. Leung WK, Wu MS, Kakugawa Y, et al. Screening for gastric cancer in Asia: current evidence and practice. Lancet Oncol. 2008;9:279-87.

3. Maehara Y, Kakeji Y, Oda S, Takahashi I, Akazawa K, Sugimachi K. Time trends of surgical treatment and the prognosis for Japanese patients with gastric cancer. $\mathrm{Br} \mathrm{J}$ Cancer. 2000;83:986-91.

4. Kitano S, Iso Y, Moriyama M, Sugimachi K. Laparoscopyassisted Billroth I gastrectomy. Surg Laparosc Endosc. 1994;4:146-8.

5. Katai H, Sasako M, Fukuda H, et al. Safety and feasibility of laparoscopy-assisted distal gastrectomy with suprapancreatic nodal dissection for clinical stage I gastric cancer: a multicenter phase II trial (JCOG 0703). Gastric Cancer. 2010;13:238-44.

6. Ohtani H, Tamamori Y, Noguchi K, et al. Meta-analysis of laparoscopy-assisted and open distal gastrectomy for gastric cancer. J Surg Res. 2011;171:479-85.

7. Kojima K, Yamada H, Inokuchi M, Kawano T, Sugihara K. A comparison of Roux-en-Y and Billroth-I reconstruction after laparoscopy-assisted distal gastrectomy. Ann Surg. 2008;247:962-7.

8. Inokuchi M, Kojima K, Yamada H, et al. Long-term outcomes of Roux-en-Y and Billroth-I reconstruction after laparoscopic distal gastrectomy. Gastric Cancer 2011.

9. Gordon AC, Kojima K, Inokuchi M, Kato K, Sugihara K. Longterm comparison of laparoscopy-assisted distal gastrectomy and open distal gastrectomy in advanced gastric cancer. Surg Endosc 2012.

10. Japanese classification of gastric carcinoma: 3rd English edition. Gastric Cancer 2011;14:101-12.

11. Japanese gastric cancer treatment guidelines 2010 (ver. 3). Gastric Cancer 2011;14:113-23.

12. Ahmed AR, Rickards G, Husain S, Johnson J, Boss T, O’Malley W. Trends in internal hernia incidence after laparoscopic Rouxen-Y gastric bypass. Obes Surg. 2007;17:1563-6.

13. Nandipati KC, Lin E, Husain F, Srinivasan J, Sweeney JF, Davis SS. Counterclockwise rotation of Roux-en-Y limb significantly reduces internal herniation in laparoscopic Roux-en-Y gastric bypass (LRYGB). J Gastrointest Surg. 2012;16:675-81.

14. Adachi Y, Shiraishi N, Kitano S. Modern treatment of early gastric cancer: review of the Japanese experience. Dig Surg. 2002;19:333-9. 
15. Shiraishi N, Adachi Y, Kitano S, Bandoh T, Katsuta T, Morimoto A. Indication for and outcome of laparoscopy-assisted Billroth I gastrectomy. Br J Surg. 1999;86:541-4.

16. Kim BJ, O’Connell T. Gastroduodenostomy after gastric resection for cancer. Am Surg. 1999;65:905-7.

17. Fujiwara M, Kodera Y, Kasai Y, et al. Laparoscopy-assisted distal gastrectomy with systemic lymph node dissection for early gastric carcinoma: a review of 43 cases. J Am Coll Surg. 2003;196:75-81.

18. Miedema BW, Kelly KA. The Roux operation for postgastrectomy syndromes. Am J Surg. 1991;161:256-61.

19. Fukuhara K, Osugi H, Takada N, Takemura M, Higashino M, Kinoshita H. Reconstructive procedure after distal gastrectomy for gastric cancer that best prevents duodenogastroesophageal reflux. World J Surg. 2002;26:1452-7.

20. Shinoto K, Ochiai T, Suzuki T, Okazumi S, Ozaki M. Effectiveness of Roux-en-Y reconstruction after distal gastrectomy based on an assessment of biliary kinetics. Surg Today. 2003;33:169-77.

21. Kubo M, Sasako M, Gotoda T, et al. Endoscopic evaluation of the remnant stomach after gastrectomy: proposal for a new classification. Gastric Cancer. 2002;5:83-9.

22. Park JM, Jin SH, Lee SR, et al. Complications with laparoscopically assisted gastrectomy: multivariate analysis of 300 consecutive cases. Surg Endosc. 2008;22:2133-9.

23. Song KY, Park $\mathrm{CH}$, Kang $\mathrm{HC}$, et al. Is totally laparoscopic gastrectomy less invasive than laparoscopy-assisted gastrectomy?: prospective, multicenter study. J Gastrointest Surg. 2008;12:1015-21.

24. Kim MG, Kawada H, Kim BS, et al. A totally laparoscopic distal gastrectomy with gastroduodenostomy (TLDG) for improvement of the early surgical outcomes in high BMI patients. Surg Endosc. 2011;25:1076-82.

25. Takaori K, Nomura E, Mabuchi $\mathrm{H}$, et al. A secure technique of intracorporeal Roux-Y reconstruction after laparoscopic distal gastrectomy. Am J Surg. 2005;189:178-83.
26. Bouras G, Lee SW, Nomura E, et al. Surgical outcomes from laparoscopic distal gastrectomy and Roux-en-Y reconstruction: evolution in a totally intracorporeal technique. Surg Laparosc Endosc Percutan Tech. 2011;21:37-41.

27. Uyama I, Sakurai Y, Komori Y, et al. Laparoscopy-assisted uncut Roux-en-Y operation after distal gastrectomy for gastric cancer. Gastric Cancer. 2005;8:253-7.

28. Morrison P, Miedema BW, Kohler L, Kelly KA. Electrical dysrhythmias in the Roux jejunal limb: cause and treatment. Am J Surg. 1990;160:252-6.

29. Miedema BW, Kelly KA. The Roux stasis syndrome. Treatment by pacing and prevention by use of an 'uncut' Roux limb. Arch Surg. 1992;127:295-300.

30. Van Stiegmann G, Goff JS. An alternative to Roux-en-Y for treatment of bile reflux gastritis. Surg Gynecol Obstet. 1988;166:69-70.

31. Noh SM. Improvement of the Roux limb function using a new type of "uncut Roux" limb. Am J Surg. 2000;180:37-40.

32. Mathias JR, Fernandez A, Sninsky CA, Clench MH, Davis RH. Nausea, vomiting, and abdominal pain after Roux-en-Y anastomosis: motility of the jejunal limb. Gastroenterology. 1985;88:101-7.

33. Gustavsson S, Ilstrup DM, Morrison P, Kelly KA. Roux-Y stasis syndrome after gastrectomy. Am J Surg. 1988;155:490-4.

34. Tu BN, Sarr MG, Kelly KA. Early clinical results with the uncut Roux reconstruction after gastrectomy: limitations of the stapling technique. Am J Surg. 1995;170:262-4.

35. Sardinas C, Gattorno F. Evaluation of gastric emptying with the "uncut" Roux en Y technique. Ann Ital Chir. 1998;69:41-6 discussion 46-7.

36. Morton JM, Lucktong TA, Trasti S, Farrell TM. Bovine pericardium buttress limits recanalization of the uncut Roux-en-Y in a porcine model. J Gastrointest Surg. 2004;8:127-31. 